\title{
A sala de aula invertida na aprendizagem do exame clínico
}

\author{
Flipped classroom for learning clinical examination
}

\author{
Rodrigo de Lemos Soares Patriota' (D) |rodrigo.patriota@gmail.com \\ Luísa Gonçalves de Frias' ${ }^{1}$ (D) luisagfrias21@gmail.com \\ Guilherme Afonso Ferreira Coelho Silton ${ }^{1}$ (D) guilherme.silton@hotmail.com \\ Marília Araújo da Silva' (D) mariliaaraujo483@gmail.com \\ Taciana Barbosa Duque ${ }^{1,2}$ (D) tacianaduque@fps.edu.br \\ Suélem Barros de Lorena1,2 (D) suelem.barros@fps.edu.br
}

\begin{abstract}
RESUMO
Introdução: A sala de aula invertida (SAI) é uma metodologia ativa de aprendizagem caracterizada pelo envio antecipado de materiais didáticos aos estudantes, de modo que o momento sala de aula seja inteiramente dedicado a atividades não expositivas. A SAl foi implementada em 2019 para aprendizagem do exame clínico $(E C)$ de alunos da graduação de Medicina em uma instituição de ensino superior da Região Nordeste do Brasil.

Objetivo: Este estudo teve como objetivo analisar a implantação da SAI na aprendizagem do EC comparando com a metodologia de miniexposição seguida de prática.

Método: Trata-se de análise de intervenção educacional com controle histórico sobre a implantação da metodologia da SAI realizada em três fases. Na primeira, os dois tutores envolvidos na implantação ou que atuaram como docentes foram avaliados por meio de entrevista semiestruturada sobre o processo de implantação da SAI e seu funcionamento inicial. A segunda fase foi a avaliação de 44 estudantes de Medicina, por meio de questionário Likert, sobre a aprendizagem com a nova metodologia. A terceira consistiu na avaliação das notas obtidas por 66 estudantes que vivenciaram o aprendizado com SAl em relação aos 142 discentes que vivenciaram a metodologia anterior.

Resultado: Os tutores avaliados conheciam pouco sobre a metodologia antes da implantação e acreditam que sua implantação promoveu ganhos, como uma maior dedicação dos estudantes ao estudo individual. A dificuldade inicial decorreu da elaboração de um extenso banco de questões para o pré-teste que era realizado no início dos momentos presenciais. Os estudantes avaliados relataram que estavam bem adaptados e que concordavam com os benefícios da SAI, como: sentir-se estimulado a estudar, desenvolver a prática com mais facilidade e a presença dos prétestes que ajudam a aprimorar o estudo individual. Os resultados da comparação das notas mostraram um aumento significativo no desempenho ao compararem os estudantes que vivenciaram a SAI $(9,11$ - DP 0,45) com os que vivenciaram a metodologia anterior $(8,49$ - DP 0,91).
\end{abstract}

Conclusão: A metodologia foi implantada de maneira satisfatória, promoveu ganhos na aprendizagem e otimizou o momento presencial para ser totalmente dedicado à aprendizagem prática.

Palavras-chave: Exame Físico; Educação Médica; Aprendizagem; Anamnese; Metodologia.

\section{ABSTRACT}

Introduction: Flipped Classroom (FC) is an Active Learning Methodology characterized by the sending of teaching materials to students in advance, so that the classroom moment is entirely dedicated to non-expository activities. The FC was implemented in 2019 for the teaching of Clinical Examination (CE) aimed at undergraduate medical students at a Higher Education Institution in the Northeast region of Brazil.

Objective: To analyze the implementation of FC for CE learning compared to the mini-exposure methodology followed by practice.

Method: Analysis of educational intervention with historical control over the implementation of the FC methodology carried out in three phases. In the first, the two tutors involved in the implementation or who acted as teachers were evaluated through a semi-structured interview about the FC implementation process and its initial operation. The second consisted in the assessment of 44 medical students, through a Likert questionnaire, on learning with the new methodology. The third consisted in evaluating the grades obtained by 66 students who experienced learning with FC in relation to 142 students who experienced the previous methodology.

Result: The evaluated tutors knew little about the methodology before it was implemented and believe that its implementation promoted gains, such as students' greater dedication to individual study. The initial difficulty arose from the creation of an extensive database of questions for the pre-test, which was carried out at the beginning of the in-person moments. The evaluated students reported being well adapted and agree with the benefits of FC, including: feeling stimulated to study, developing the practice more easily and the presence of pre-tests that help to improve individual study. The results of the comparison of grades showed a significant increase in performance when comparing students who experienced the FC $(9,11-S D 0,45)$ with those who experienced the previous methodology $(8,49-S D 0,91)$.

Conclusion: The methodology was satisfactorily implemented, promoted gains in learning and optimized the in-person time to be entirely dedicated to practical learning.

Keywords: Physical Examination; Medical Education; Learning; Anamnesis; Methodology.

${ }^{1}$ Faculdade Pernambucana de Saúde, Recife, Pernambuco, Brasil.

${ }^{2}$ Instituto de Medicina Integral Professor Fernando Figueira, Recife, Pernambuco, Brasil.

Editora-chefe: Rosiane Viana Zuza Diniz. Editor associado: Kristopherson Lustosa Augusto.

Recebido em 06/09/21; Aceito em 17/11/21. | Avaliado pelo processo de double blind review. 


\section{INTRODUÇÃO}

O método tradicional de aprendizagem em que o professor e o conteúdo ministrado são os protagonistas do processo ensino-aprendizagem vem sendo desafiado pelas novas metodologias de ensino, cujo centro do processo é o discente. Durante séculos, estudantes permaneceram em silêncio, em cadeiras enfileiradas, escutando atentamente os conteúdos repassados pelos professores, os donos do conhecimento do qual os alunos ainda não eram detentores ${ }^{1}$.

Entretanto, as metodologias tradicionais se mostraram menos eficazes na aprendizagem de atitudes e habilidades para as novas gerações, cujo acesso à informação é de larga escala e que, diante de todas as distrações do mundo contemporâneo, sentem dificuldades em manter a concentração necessária para entendimento das aulas expositivas. Além disso, o protagonismo na aprendizagem deveria ser dado à parte mais interessada, que é o estudante ${ }^{2}$.

Nesse contexto, algumas técnicas mais inovadoras de ensino surgem, chamadas de forma mais ampla de metodologias ativas, em que o estudante se torna mais ativo no processo de ensino-aprendizagem, com eficácia demonstrada para o ensino de disciplinas do ensino médio e do ensino superior ${ }^{3-5}$.

Entre essas metodologias, há a sala de aula invertida (SAI), do inglês flipped classroom, que promove uma modificação na sequência no processo de aprendizagem, uma vez que o conteúdo, em vez de ser ministrado na forma de exposição em sala de aula, é aprendido pelo estudante previamente, por meio de material audiovisual e escrito. No momento presencial, o conteúdo previamente estudado pode ser compartilhado e avaliado de diversas formas, como debates, jogos de perguntas, experimentos e apresentações orais ${ }^{6-8}$.

A SAl tem sido aplicada no ensino da medicina com bons resultados, tanto na graduação como na pós-graduação, demonstrando ganhos na aprendizagem de conteúdos teóricos e práticos em laboratórios de ensino de habilidades procedurais ${ }^{9-13}$.

A aprendizagem de habilidades, competências e atitudes envolvidas no exame clínico, por exemplo, necessita de treinamentos que costumam ser realizados em laboratórios de prática simulada. Esses procedimentos vão além de uma formação técnica e caracterizam-se por ser o primeiro contato com a relação médico-paciente ${ }^{14,15}$.

Nesse contexto, a SAI foi implantada para aprendizagem do exame clínico em uma escola médica do Nordeste. Seu processo de implantação e seu impacto são objetos de análise neste estudo.

\section{METODOLOGIA}

Realizou-se análise de intervenção educacional com controle histórico sobre a implantação da metodologia de SAI. Em 2020, o estudo foi desenvolvido na Faculdade Pernambucana de Saúde (FPS), em três etapas.

A primeira etapa consistiu em entrevista semiestruturada individual, com perguntas previamente selecionadas, com os dois tutores que participaram do processo de implantação da SAI no ensino do exame clínico, sendo um deles o atual docente responsável pela área de conhecimento na instituição. A seleção dos tutores ocorreu por conveniência. A escolha do método se deu em virtude de promover a coleta de mais detalhes do processo, de forma mais flexível e abrangente. As entrevistas foram realizadas com perguntas amplas com o intuito de entender como se deu a implantação da SAI na aprendizagem do exame clínico no curso de Medicina da FPS e seu impacto atual na aprendizagem. As entrevistas foram gravadas em áudio, com a autorização dos participantes ${ }^{16}$.

O segundo passo foi realizado com a aplicação de um survey sobre a utilização da SAI no ensino do exame clínico, para os estudantes que vivenciaram a aprendizagem da área de conhecimento por essa metodologia. Apresentou-se a pesquisa, em sala de aula, aos 66 estudantes, e realizou-se o convite para participar do estudo. A amostra foi obtida por conveniência. Quarenta e quatro estudantes responderam, de modo digital, a um formulário dividido em dois blocos: o primeiro para coleta de características sociodemográficas, conhecimento e experiência com metodologias ativas de aprendizagem, e o segundo bloco estruturado em escala Likert de cinco pontos contendo assertivas baseadas na vivência com a SAI.

$\mathrm{Na}$ terceira etapa, coletaram-se informações sobre o desempenho de 208 estudantes que desenvolveram de aprendizagem do exame clínico, dentro da metodologia de SAI (66), em 2019, e da metodologia anterior (142), em 2018, por meio dos resultados das avaliações. Informações foram obtidas por meio do registro acadêmico da escola médica sobre as notas dos participantes nas avaliações formais do semestre letivo.

$\mathrm{Na}$ primeira etapa, as entrevistas foram transcritas e analisadas de acordo com as respostas obtidas em cada pergunta realizada. As respostas foram agrupadas em citações diretas e sumarizadas em quadros. Posteriormente, elaborou-se uma narrativa baseada nesses dados.

Para a avaliação do survey realizado com os estudantes, os dados coletados foram armazenados e organizados em planilha Excel ${ }^{\circledR}$, versão 2013, com dupla entrada de dados com conferência e verificação de erros de digitação. O programa utilizado para a análise estatística foi o Epilnfo ${ }^{\circledR}$ versão 7.1. Para a análise dos dados, obtiveram-se inicialmente as medidas de tendência central e dispersão para as variáveis contínuas e medidas de distribuição de frequência para as categóricas. Para a análise da opinião dos estudantes na escala Likert, adotouse como critério de consenso o valor do ranking médio (RM) 
acima de 3,0 como concordância. Para análise da consistência do formulário, utilizou-se o alfa de Cronbach.

As notas obtidas pelos estudantes que vivenciaram a SAI foram comparadas com as notas obtidas pelos estudantes que não vivenciaram; estas foram e coletadas e organizadas em planilha Excel $^{\circledR}$ versão 2013 e dispostas em médias e desvios padrão. Utilizou-se o teste estatístico de Kruskal-Wallis, considerando um nível de significância de $p<0,05$ e intervalo de confiança (IC) de 95\%, por meio do programa estatístico Epilnfo $^{\circledR}$ versão 7.1 .

O projeto foi aprovado pelo Comitê de Ética da FPS: Certificado de Apresentação para Apreciação Ética (CAAE) no 26405319.0.0000.5569.

\section{RESULTADOS}

\section{Entrevista semiestruturada com tutores}

Realizaram-se entrevistas com dois docentes da FPS. O primeiro entrevistado (A) participou ativamente do processo de implantação da SAI e exercia a docência na metodologia anterior à implantação; o segundo entrevistado (B) participou ativamente da implantação e é o atual docente da área de conhecimento. Ambos não tinham contato prático com a metodologia antes da implantação, mas a conheciam em teoria e tinham experiência com outras metodologias ativas de aprendizagem.

Os entrevistados relataram que a implantação surgiu de dificuldades encontradas na aprendizagem do exame clínico, pois, como antes era baseada em miniexposições seguidas de práticas, o tempo das exposições reduzia bastante o tempo de prática. Com a mudança na metodologia de ensino, os objetivos de aprendizagem foram modificados, e o módulo começou a integrar conteúdos teóricos e práticos. O modelo de ensino implementado foi baseado no envio prévio de materiais (vídeos, textos e diapositivos) e em um momento presencial, que se inicia com um pré-teste seguido de atividade de aprendizagem em laboratório de simulação prática.

A necessidade do pré-teste a cada encontro foi uma das dificuldades encontradas no início do processo, pois, para sua aplicação, houve a necessidade de elaborar um extenso banco de questões capazes de estimular o raciocínio clínico do estudante e não apenas a sua capacidade de memorização. Utilizou-se a ferramenta digital disponível em http://www. quizes.com para armazenar as questões construídas e facilitar o processo de correção.

Em relação à participação dos estudantes nas atividades de aprendizagem, os entrevistados relatam que os alunos estão mais participativos e se dedicam mais ao estudo individual que anteriormente. A presença dos minitestes deixou os estudantes mais ansiosos durante o período inicial de adaptação ao novo método, o que provocou impacto em notas mais baixas nos pré-testes e inibiu a participação dos estudantes nas atividades práticas, mas essas dificuldades logo foram superadas.

Uma dificuldade apontada pelos tutores avaliados foi em relação aos estudantes monitores, que realizavam sua atividade por meio de exposições sobre a matéria de estudo. Com a nova metodologia, em que as exposições deixaram de ser realizadas, esse modelo de monitoria se tornou não coerente com o modelo. Apesar das orientações iniciais, os monitores ainda persistiram realizando sua atividade da maneira anterior, dificuldade que só conseguiu ter resolução após os docentes realizarem exaustiva orientação sobre o método e, posteriormente, com a seleção de novos monitores que já foram admitidos dentro de uma nova perspectiva.

Para o entrevistado A: "No fundamento, a metodologia é muito interessante, porque, caso o aluno que se interesse pelo recurso, que estuda em casa, participa ativamente da monitoria, chega no encontro, faz o miniteste que avalia seus conhecimentos e vai praticar, para esse o fundamento é muito interessante". De acordo com o entrevistado: "É uma metodologia de ensino ativa, muito boa. Eu sinto hoje uma satisfação muito grande de estar trabalhando nesse formato".

\section{Pesquisa com os estudantes}

O total de participantes do estudo foi de 44 discentes do primeiro ano do curso de Medicina da FPS, correspondendo a uma taxa de resposta de cerca de $66,6 \%$. A idade variou entre 17 e 46 anos, predominando a faixa etária menor que 25 anos. O sexo feminino prevaleceu na amostra (75\%). Cerca de $20 \%$ estudaram em outra faculdade, dos quais quatro (9\%) concluíram outra graduação. Em relação à utilização de metodologia ativas antes de ingressarem na FPS, cerca de $90 \%$ nunca a utilizaram, e, apesar de 28 estudantes (60\%) informarem ter conhecimento da metodologia de SAI, apenas três referiram tê-la utilizado previamente (Tabela 1).

Sobre a opinião dos estudantes quanto à utilização da metodologia de SAl, os resultados foram agrupados em três blocos: assertivas sobre a preparação, orientação e adaptação, assertivas sobre atitude em relação ao estudo e processo de aprendizagem e assertivas sobre a utilização do pré-teste (Tabela 2). De modo geral, os discentes enxergam positivamente a implantação da SAI na aprendizagem do exame clínico, com alfa de Cronbach de 0,87.

No primeiro bloco, os estudantes concordam que receberam explicações sobre o método (RM4,27 $\pm 1,54)$, entendem o papel do tutor (RM 4,57 $\pm 1,82$ ) e compreendem o método (RM $4,55 \pm 1,73$ ). No segundo bloco, os estudantes afirmam estudar regularmente antes das aulas (RM 3,91 $\pm 1,45$ ) e, principalmente, pelas fontes enviadas pelo tutor (RM4,20 $\pm 1,44)$. No terceiro bloco, 
os pesquisados aprovam o pré-teste, uma vez que discordam que o pré-teste contribui pouco para o aprendizado ( $\operatorname{RM} 2,39 \pm 1,18)$, não possibilita mostrar o conhecimento que possuem (RM 2,11 $\pm 1,34)$, é cansativo ( $\operatorname{RM} 2,27 \pm 1,32$ ) e não ajuda na construção do conhecimento (RM 2,05 $\pm 1,38$ ). Os estudantes consideram que o pré-teste orienta os estudos antes do momento presencial, já que discordam que o pré-teste não influencia a forma com estudam $(2,30 \pm 1,29)$ e que respondem ao pré-teste apenas com conhecimentos prévios $(2,07 \pm 1,37)$, assim como concordam que, com o pré-teste, sentem-se mais estimulados a estudar antes da atividade prática (RM 3,82 $\pm 1,19)$.

\section{Avaliação das notas}

As notas obtidas pelos estudantes que vivenciaram o ensino do exame clínico antes e depois da implantação da SAI estão expostas na tabela 3. O processo de avaliação nos dois momentos foi semelhante, exceto pela presença dos pré-testes, que foi introduzido após a implantação da SAI.

Tabela 1. Características sociodemográficas dos estudantes de Medicina que avaliaram o método de sala de aula invertida no estudo do exame clínico - FPS, 2020.

\begin{tabular}{|c|c|c|}
\hline Características sociodemográficas & $\mathrm{N}(44)$ & $\%$ \\
\hline \multicolumn{3}{|l|}{ Idade } \\
\hline$<25$ anos & 39 & 88,63 \\
\hline $25-30$ anos & 3 & 6,81 \\
\hline $30-40$ anos & 2 & 4,56 \\
\hline \multicolumn{3}{|l|}{ Sexo } \\
\hline Feminino & 31 & 70,45 \\
\hline Masculino & 13 & 29,55 \\
\hline \multicolumn{3}{|l|}{ Já estudou em outra faculdade? } \\
\hline Sim & 9 & 20,45 \\
\hline Não & 35 & 79,55 \\
\hline \multicolumn{3}{|l|}{ Possui outra graduação? } \\
\hline $\operatorname{Sim}$ & 4 & 9,09 \\
\hline Não & 40 & 90,91 \\
\hline \multicolumn{3}{|c|}{$\begin{array}{l}\text { Já utilizou alguma metodologia ativa antes de ingressar na } \\
\text { Faculdade Pernambucana de Saúde? }\end{array}$} \\
\hline Sim & 2 & 4,55 \\
\hline Não & 42 & 95,45 \\
\hline \multicolumn{3}{|c|}{$\begin{array}{l}\text { Já utilizou a sala de aula invertida como método de aprendizado } \\
\text { antes de ingressar na Faculdade Pernambucana de Saúde? }\end{array}$} \\
\hline Sim & 3 & 6,82 \\
\hline Não & 41 & 93,18 \\
\hline \multicolumn{3}{|c|}{$\begin{array}{l}\text { Tinha conhecimento do método da sala de aula invertida } \\
\text { antes de ingressar na Faculdade Pernambucana de Saúde? }\end{array}$} \\
\hline Sim & 28 & 63,64 \\
\hline Não & 16 & 36,36 \\
\hline
\end{tabular}

Tabela 2. Opinião de estudantes do primeiro ano do curso de Medicina sobre a utilização da metodologia de sala de aula invertida no estudo do exame clínico em ambientes simulados - FPS, 2020.

\section{Assertivas}

$R M^{\mathrm{a}} \pm(\mathrm{DP})^{\mathrm{b}}$

BLOCO 1: Preparação, orientação e adaptação à metodologia

Eu recebi explicação sobre o método de sala de aula invertida.

$4,27 \pm 1,54$

Eu entendo o papel do tutor no método da sala de aula invertida.

$4,57 \pm 1,82$

Eu compreendo o método de sala de aula invertida.

$4,55 \pm 1,73$

Eu recebo o material para leitura antes dos encontros.

$3,11 \pm 0,96$

O tutor está disponível para tirar dúvidas.

$3,95 \pm 1,18$

Eu estou bem adaptado ao método da sala de aula invertida.

$3,43 \pm 1,30$

Eu gosto do método de sala de aula invertida.

$3,77 \pm 1,13$

BLOCO 2: Atitude em relação ao estudo

Eu estudo regularmente antes dos encontros em sala.

$3,91 \pm 1,45$

Eu estudo por livro antes dos encontros.

$4,39 \pm 1,59$

Eu estudo por videoaulas antes dos encontros.

$3,48 \pm 1,05$

Eu estudo pelas fontes que são enviadas pelo tutor.

$4,20 \pm 1,44$

Eu busco outras fontes além das indicadas pelo tutor.

$3,66 \pm 1,11$

Eu estudo antes dos encontros em sala com os monitores.

$3,43 \pm 1,08$

A metodologia de sala de aula invertida me estimula a estudar mais.

$3,70 \pm 1,05$

Eu desenvolvo a parte prática da aula com facilidade devido ao estudo prévio.

$3,77 \pm 1,22$

A metodologia da sala de aula invertida me ajuda a fixar o conhecimento.

$3,70 \pm 1,31$

BLOCO 3: Utilização de pré-teste associada à metodologia de sala de aula invertida

Uso o pré-teste para guiar os meus estudos.

$3,27 \pm 0,93$

Eu estudo antes da aula apenas pela existência do pré-teste.

$2,84 \pm 1,05$

Eu uso mais da minha capacidade de memorização para responder o pré-teste.

$3,50 \pm 1,0$

Eu respondo o pré-teste apenas com meus conhecimentos prévios.

$2,07 \pm 1,37$

O pré-teste não influencia na forma como estudo.

$2,30 \pm 1,29$

Eu considero o pré-teste um importante elemento para minha formação acadêmica. 
Tabela 2. (Continuação) Opinião de estudantes do primeiro ano do curso de Medicina sobre a utilização da metodologia de sala de aula invertida no estudo do exame clínico em ambientes simulados - FPS, 2020.

\begin{tabular}{l} 
Assertivas \\
\hline BLOCO 3: Utilização de pré-teste associada à \\
sala de aula invertida \\
Eu considero que o pré-teste contém uma \\
quantidade adequada de questões.
\end{tabular}

Eu considero que o pré-teste possui um nível de dificuldade adequado.

Eu considero as questões do pré-teste bem elaboradas.

Eu considero que o tempo para responder o pré-teste é suficiente.

$R M^{\mathrm{a}} \pm(\mathrm{DP})^{\mathrm{b}}$

Com o pré-teste me sinto mais estimulado a estudar antes da atividade prática.

Se o pré-teste não existisse, eu estudaria menos para atividades de semiologia.

O pré-teste me ajuda a fixar o que aprendi.

O feedback após o pré-teste me ajuda a construir melhor meu conhecimento.

Eu considero que o pré-teste contribui pouco para o meu aprendizado.

O pré-teste não possibilita mostrar o conhecimento que possuo.

$3,43 \pm 1,04$

$3,61 \pm 1,29$

$3,57 \pm 1,24$

$3,98 \pm 1,29$

$3,89 \pm 1,19$

$3,34 \pm 0,94$

$3,57 \pm 1,14$

$3,82 \pm 1,19$

$2,39 \pm 1,18$

$2,11 \pm 1,34$

Eu considero o pré-teste cansativo.

$2,27 \pm 1,32$

Eu considero que o pré-teste não ajuda na construção do meu conhecimento.

$2,05 \pm 1,38$

${ }^{\mathrm{a}} \mathrm{RM}=$ ranking médio; $^{\mathrm{b}} \mathrm{DP}=$ desvio padrão.

Tabela 3. Média obtida nas avaliações de estudantes de Medicina que vivenciaram a aprendizagem do exame clínico com a metodologia SAI comparando com controle histórico imediatamente anterior de turma que não utilizou a SAI.

\begin{tabular}{|c|c|c|c|}
\hline & \multicolumn{2}{|c|}{ Sala de aula invertida } & \multirow{2}{*}{$\begin{array}{c}\text { Análise } \\
\text { estatística }\end{array}$} \\
\hline & Não (n 142) & $\operatorname{Sim}(n 66)$ & \\
\hline Média (DP) & $8,4939(0,9133)$ & $9,1062(0,4479)$ & $P=0,000$ \\
\hline
\end{tabular}

${ }^{a}$ Kruskall-Wallis.

A média das notas dos 66 estudantes que vivenciaram a SAI $(9,102$ - DP 0,4479) foi maior quando comparada com a obtida pelos 142 que não vivenciaram a SAI (8,4939 - DP 0,9133). A nota mais baixa obtida pelos estudantes da metodologia anterior foi 6,370, e, com a SAI, os discentes obtiveram 7,660 (Tabela 3).

\section{DISCUSSÃO}

A utilização da SAI para a aprendizagem de habilidades, competências e atitudes no curso de Medicina tem demonstrado ser bastante efetiva e bem-aceita por docentes e discentes, achados que foram encontrados também no nosso estudo ${ }^{11,12}$.

A pouca experiência prévia de docentes e discentes com a SAI evidenciada na entrevista com os docentes e vista na pesquisa com discentes não foi um impeditivo para a implantação da SAI na aprendizagem do exame clínico. Essa dificuldade foi superada, sobretudo, pelo conhecimento teórico dos tutores sobre o assunto e pela experiência com outras metodologias ativas de aprendizagem. Jakobsen et al. ${ }^{17}$ propõem o uso da Aprendizagem Baseada em Times (Team Based Learning - TBL), que é uma metodologia mais difundida, como forma de contribuir no processo de estruturação da SAI. Oliveira et al. utilizaram-se da mesma estratégia em um curso de expansão do ensino médico no Brasil com bons resultados ${ }^{18}$.

Apontou-se a presença do pré-teste como uma dificuldade inicial, principalmente na sua operacionalização, embora na literatura pesquisada não haja menção a essa dificuldade. O pré-teste é usado com frequência nas experiências de outras escolas médicas que adotam a SAl, inclusive associado a um pós-teste para aferir a retenção de conteúdos. A presença de quizzes como forma de pré-teste foi avaliada em metanálise, e verificou-se que, quando utilizados no início das aulas, tornam a aplicação da SAI na aprendizagem de profissionais da área de saúde mais efetiva ${ }^{10,13,19}$.

Os estudantes relataram que o pré-teste é uma ferramenta útil no processo de aprendizagem, e sua presença pode direcionar o estudo individual. Os docentes apontaram que, inicialmente, os estudantes se mostraram mais ansiosos com a presença do pré-teste e que, com o tempo, superaram essa dificuldade inicial. Em contraste com nosso achado, Uchida et al. ${ }^{20}$ compararam a SAI com a metodologia tradicional para o ensino de uma manobra semiológica e constataram que os estudantes que vivenciaram a SAI se mostraram mais autoconfiantes na realização do pré-teste em sala de aula do que aqueles que vivenciaram a metodologia tradicional.

A dificuldade encontrada em relação à readaptação da forma como os estudantes monitores deveriam atuar no processo de aprendizagem, quando olhada retrospectivamente, foi superada, e não se encontrou problema semelhante na literatura. No nosso estudo, constatou-se a dedicação dos tutores em resolver esse entrave com os estudantes monitores devido à importância destes no processo de aprendizagem, como observado por Nunes et al. ${ }^{21}$.

A SAI foi bem-aceita por parte dos estudantes, aspecto observado tanto na pesquisa que envolveu os estudantes 
quanto na percepção dos docentes. Na literatura9,10,18,22, verifica-se que a metodologia, de modo geral, é bem-aceita pelos estudantes, exceto por Sajid et al. ${ }^{23}$ que constataram que os estudantes avaliados não tiveram boa percepção da metodologia nem maior retenção de conteúdo ${ }^{23}$.

No nosso trabalho, os estudantes que tiveram a experiência com a SAI obtiveram melhores notas quando comparados aos que não tiveram. Metanálise publicada por Chen et al. ${ }^{24}$ reporta a presença de estudos com resultados positivos e neutros. Em Martinelli et al. ${ }^{19}$, os estudantes preferiram a SAI a outras metodologias, apesar de não ter havido superioridade em relação à retenção de conhecimento.

\section{CONCLUSÃO}

Pela análise realizada em nosso estudo, a implantação da SAI na aprendizagem do exame clínico ocorreu de forma satisfatória e promoveu ganhos consideráveis na construção do conhecimento, visto pela avaliação de docentes e discentes, o que foi corroborado pela elevação das médias obtidas no módulo.

A metodologia pode ser aplicada em outras áreas de conhecimento que tenham necessidades semelhantes em relação à otimização dos momentos presenciais a serem dedicados à aprendizagem prática, podendo ser fruto de novos estudos de intervenção educacional e análise.

\section{CONTRIBUIÇÃO DOS AUTORES}

Os autores participaram de todas as etapas do estudo e da redação do artigo.

\section{CONFLITO DE INTERESSES}

Declaramos não haver conflito de interesses.

\section{FINANCIAMENTO}

Declaramos não haver financiamento.

\section{REFERÊNCIAS}

1. Keiler LS. Teachers' roles and identities in student-centered classrooms. Int J STEM Educ. 2018;5(1):34

2. Gillispie V. Using the flipped classroom to bridge the gap to generation Y. Ochsner J. 2016;16(1):32-6.

3. Freeman S, Eddy SL, McDonough M, Smith MK, Okoroafor N, Jordt H, et al. Active learning increases student performance in science, engineering, and mathematics. Proc Natl Acad Sci U S A. 2014;111(23):8410-5.

4. Cavanagh AJ, Chen X, Bathgate M, Frederick J, Hanauer DI, Graham MJ. Trust, growth mindset, and student commitment to active learning in a college science course. CBE Life Sci Educ. 2018;17(1):1-8.
5. Sivarajah RT, Curci NE, Johnson EM, Lam DL, Lee JT, Richardson ML. A review of innovative teaching methods. Acad Radiol. 2019;26(1):101-13.

6. McLean S, Attardi SM, Faden L, Goldszmidt M. Flipped classrooms and student learning: not just surface gains. Adv Physiol Educ. 2016;40(1):47-55.

7. Reidsema C, Kavanagh L, Hadgraft R, Smith N, editors. The flipped classroom: practice and practices in higher education. Singapore. Springer; 2017.

8. Njie-Carr VPS, Ludeman E, Lee MC, Dordunoo D, Trocky NM, Jenkins LS. An integrative review of flipped classroom teaching models in nursing education. J Prof Nurs. 2017;33(2):133-44.

9. Jung $\mathrm{H}, \mathrm{An} J$, Park KH. Analysis of satisfaction and academic achievement of medical students in a flipped class. Korean J Med Educ. 2018;30(2):101-7.

10. Hew KF, Lo CK. Flipped classroom improves student learning in health professions education: a meta-analysis. BMC Med Educ. 2018 Dec 15;18(1):38

11. Hu X, Zhang H, Song $Y$, Wu C, Yang Q, Shi Z, et al. Implementation of flipped classroom combined with problem-based learning: an approach to promote learning about hyperthyroidism in the endocrinology internship. BMC Med Educ. 2019;19(1):1-8.

12. Cheng $X$, Lee $\mathrm{KKH}$, Chang EY, Yang X. The "flipped classroom" approach: stimulating positive learning attitudes and improving mastery of histology among medical students. Anat Sci Educ. 2017;10(4):317-27.

13. Sezer B, Abay E. Looking at the Impact of the flipped classroom model in medical education. Scand J Educ Res. 2019;63(6):853-68.

14. Porto CC. Semiologia médica. 8a ed. Rio de Janeiro: Guanabara Koogan; 2019.

15. Costa GPO, Herculano TB, Gama ALH, Cabral RP, Campos DB, Oliveira DNS. Enfrentamentos do estudante na iniciação da semiologia médica. Rev Bras Educ Med. 2018;42(2):79-88.

16. Moré CL. A "entrevista em profundidade" ou "semiestruturada" no contexto da saúde. Investigação Qualitativa em Ciências Sociais. 2015;3:126-31.

17. Jakobsen K, Knetemann M. Putting structure to flipped classrooms using team-based learning. Int J Teach Learn High Educ. 2017;29(1):177-85.

18. Lucchetti AL, Ezequiel OS, Oliveira IN, Moreira-Almeida A, Lucchetti G. Using traditional or flipped classrooms to teach "Geriatrics and Gerontology"? Investigating the impact of active learning on medical students' competences. Med Teach. 2018;40(12):1248-56.

19. Martinelli SM, Chen F, DiLorenzo AN, Mayer DC, Fairbanks S, Moran K, et al. Results of a flipped classroom teaching approach in anesthesiology residents. J Grad Med Educ. 2017;9(4):485-90.

20. Uchida S, Shikino K, Ishizuka K, Yamauchi Y, Yanagita Y, Ikusaka M. The flipped classroom is effective for medical students to improve deep tendon reflex examination skills: a mixed-method study. Res Sq 2021;(Preprint):1-13.

21. Nunes GP, Pirovani BO, Silva HG, Butarelo AV, Rossini JS, Costa JM, et al. The importance of student monitoring in academic learning: a two-year follow-up. Brazilian J Oral Sci. 2018;17:1-7.

22. Fatima SS, Hashmi S, Rehman R, Akbar R. Teaching "shock pathophysiology" by flipped classroom. Pakistan J Med Sci. 2019;35(6):1631-5.

23. Sajid M, Shaikh AA, Ikram MF, Cahusac P, Yaqinuddin A, AlKattan W, et al. Comparative analysis of effectiveness between flipped classroom and lecture-based classroom in undergraduate medical education at Alfaisal University. Cureus. 2020;12(11):e11408.

24. Chen F, Lui AM, Martinelli SM. A systematic review of the effectiveness of flipped classrooms in medical education. Med Educ. 2017;51(6):585-97. 